\title{
Computational Simulations to Predict Creatine Kinase-Associated Factors: Protein-Protein Interaction Studies of Brain and Muscle Types of Creatine Kinases
}

\author{
Wei-Jiang Hu, ${ }^{1}$ Sheng-Mei Zhou, ${ }^{2}$ Joshua SungWoo Yang,, ${ }^{3}$ and Fan-Guo Meng1 \\ ${ }^{1}$ Zhejiang Provincial Key Laboratory of Applied Enzymology, Yangtze Delta Region Institute of Tsinghua University, \\ Jiaxing 314006, China \\ ${ }^{2}$ College of Biology and Chemical Engineering, Jiaxing University, Jiaxing 314001, China \\ ${ }^{3}$ Korean Bioinformation Center (KOBIC), Korea Research Institute of Bioscience \& Biotechnology (KRIBB), \\ Daejeon 305-806, Republic of Korea \\ ${ }^{4}$ Department of Bioinformatics, University of Sciences \& Technology, Daejeon 205-305, Republic of Korea
}

Correspondence should be addressed to Fan-Guo Meng, mengfanguo@tsinghua.org.cn

Received 17 May 2011; Accepted 26 May 2011

Academic Editor: Jun-Mo Yang

Copyright (c) 2011 Wei-Jiang Hu et al. This is an open access article distributed under the Creative Commons Attribution License, which permits unrestricted use, distribution, and reproduction in any medium, provided the original work is properly cited.

Creatine kinase (CK; EC 2.7.3.2) is related to several skin diseases such as psoriasis and dermatomyositis. CK is important in skin energy homeostasis because it catalyzes the reversible transfer of a phosphoryl group from MgATP to creatine. In this study, we predicted CK binding proteins via the use of bioinformatic tools such as protein-protein interaction (PPI) mappings and suggest the putative hub proteins for CK interactions. We obtained 123 proteins for brain type CK and 85 proteins for muscle type CK in the interaction networks. Among them, several hub proteins such as NFKB1, FHL2, MYOC, and ASB9 were predicted. Determination of the binding factors of CK can further promote our understanding of the roles of CK in physiological conditions.

\section{Introduction}

Creatine kinase (CK) (ATP: creatine kinase N-phosphotransferase, EC 2.7.3.2) is thought to be crucial for intracellular transport and the storage of high energy phosphate because it catalyzes the reversible transfer of a phosphoryl group from MgATP to creatine, which leads to the creation of phosphocreatine and MgADP [1]. CK plays an important role in the cellular energy metabolism of vertebrates, and it is widely distributed in tissues that require a lot of energy [2]. Several types of CK are expressed in various tissues: the muscle and brain types of $\mathrm{CK}$ are the most common, and three different isoenzymes that include CK-MM (the muscle type homodimer), CK-BB (the brain type homodimer), and CK$\mathrm{MB}$ (the muscle plus brain type heterodimer) originate from these two common types. CK is an important serum marker for myocardial infarction. Various types of CKs (the muscle, brain, and mitochondrial types) are thought to be important not only in the diagnosis of myocardial infarction, cardiac hypertrophy, and muscular dystrophy but also for studies of some other serious diseases, including Alzheimer's disease, Parkinson's disease, and psoriasis [3-8].

$\mathrm{CK}-\mathrm{BB}$ is associated with several pathologies, including neurodegenerative and age-related diseases. Recently, Chang et al. [9] reported an important role for CK-BB in osteoclast-mediated bone resorption, which was found using a proteomics approach. They found that CK-BB is greatly increased during osteoclastogenesis and suggested that it represents a potential target for antiresorptive drug development. CK-BB interacts with the potassium-chloride cotransporter 3, which is involved in the pathophysiology of hereditary motor and sensory neuropathy with agenesis of the corpus callosum [10]. Previous studies [11, 12] have reported that CK-BB is involved in Alzheimer's disease (AD) as an oxidatively modified protein. This suggests that oxidatively damaged CK-BB may be associated with aging and agerelated neurodegenerative disorders such as $\mathrm{AD}$.

CK-MM is a good model to use for studying folding pathways because of several characteristics: (i) it is a dimer 


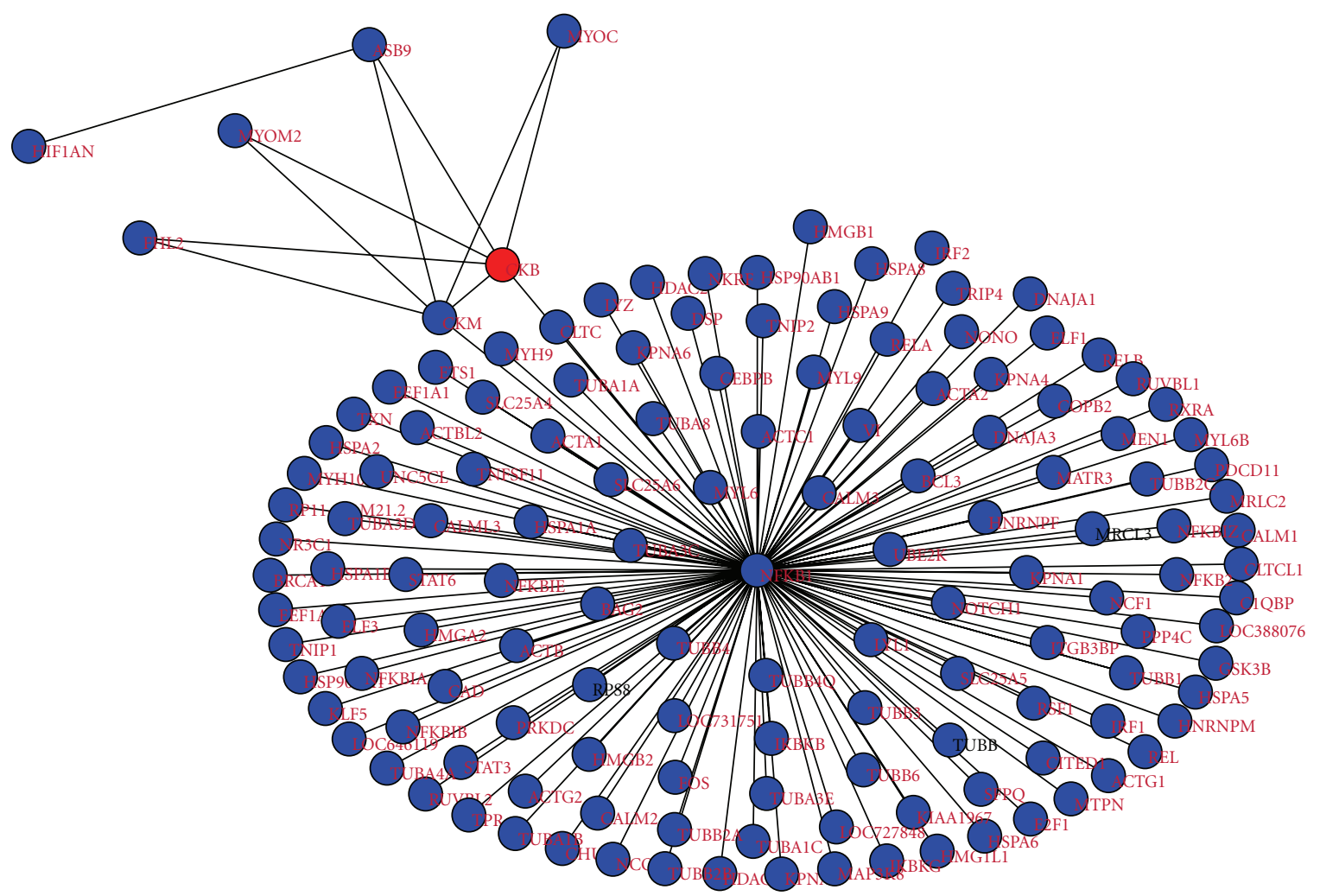

FIGURE 1: PPI map for CKB as a target hub protein with the $80 \%$ identity. Labels with red color indicate the hub protein of targeting. The image was made by the aiSee program (http://www.aisee.com/).

that consists of two identical subunits, each with an N-terminal domain with about 100 residues and a C-terminal domain with about 250 residues connected by a long linker [13]; (ii) extensively denatured CK can be renatured spontaneously with restoration of its enzymatic activity in the absence of any external assistance [14]; (iii) its folding pathway is complicated and involves several intermediates [15, 16]; (iv) conformational changes of the secondary and tertiary structures can be easily measured by monitoring activity changes $[14,15]$; (v) protein-protein interactions, including molecular chaperones, are observed during refolding [17, $18]$.

In this study, we obtained computational predictions of the binding proteins by using two types of CK (CK-BB and CK-MM) as hub proteins in bioinformatic algorithms. As a result, we obtained 208 protein lists in the interaction networks via application of both muscle and brain types of CK. Determination of the binding factors and functions of CK can further promote our understanding of the physiological roles of CK.

\section{Materials and Methods}

2.1. PPI Mappings: PEIMAP and PSIMAP Algorithms. We present the functionally classified protein-protein interactions on the basis of the cell cycle, cell transport, oxidoreductase, and apoptosis. PPI resources were assembled from a combination of several experimental protein interaction databases. The protein interaction resources included six databases: DIP [19], BIND [20], IntAct [21], MINT [22], HPRD [23], and BioGrid [24]. We performed a redundancy test to remove identical protein sequences from the interaction databases. The databases contain 116,773 proteins and 229,799 interactions.

PPI prediction uses most of the major types of PPI algorithms. They are (1) Protein Structural Interactome MAP (PSIMAP), a method that uses the structural domain of the SCOP (Structural Classification of Proteins) database [25] and (2) Protein Experimental Interactome MAP (PEIMAP), a common method that uses public resources of experimental protein interaction information such as HPRD, BIND, DIP, MINT, IntAct; and BioGrid. The basic procedure of PSIMAP is to infer interactions between proteins by using their homologs. Interactions among domains or proteins for known PDB (Protein Data Bank) structures are the basis for the prediction. If an unknown protein has a homolog to a domain, then PSIMAP assumes that the query has the probability to interact with its homolog's partners. This concept is called "homologous interaction." The original interaction between two proteins or domains is based on the Euclidean distance. Therefore, PSIMAP gives a structure-based interaction prediction [26]. PEIMAP was constructed by combining several experimental protein-protein interaction databases. We carried out a redundancy check to remove identical protein sequences from the source interaction 


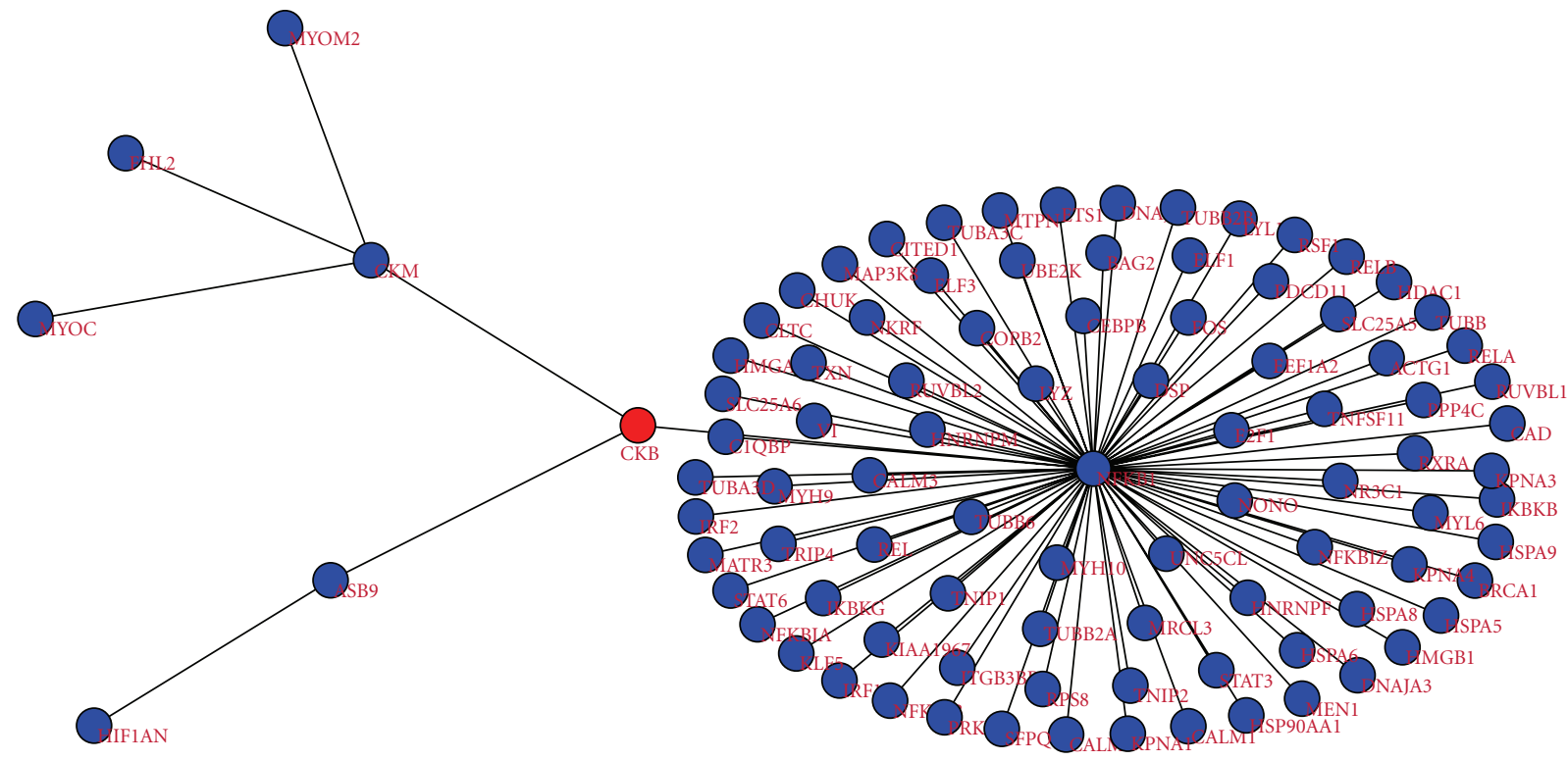

FIgURE 2: PPI map for CKB as a target hub protein with the $100 \%$ identity. The methodological conditions were the same as for Figure 1 except the identity.

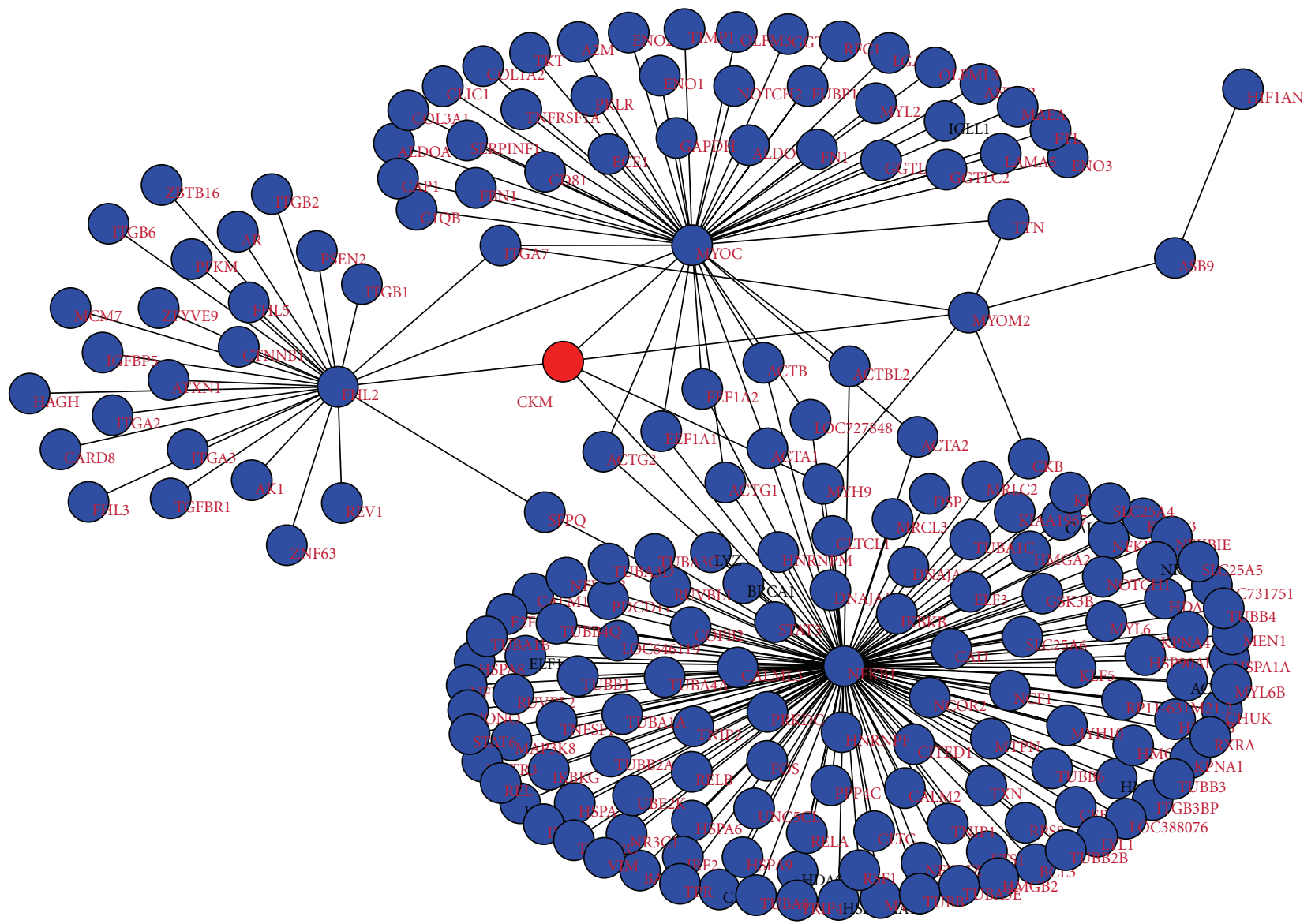

FIGURE 3: PPI map for CKM as a target hub protein with the $80 \%$ identity. Labels with red color indicate the hub protein of targeting. The image was made by the aiSee program (http://www.aisee.com/). 


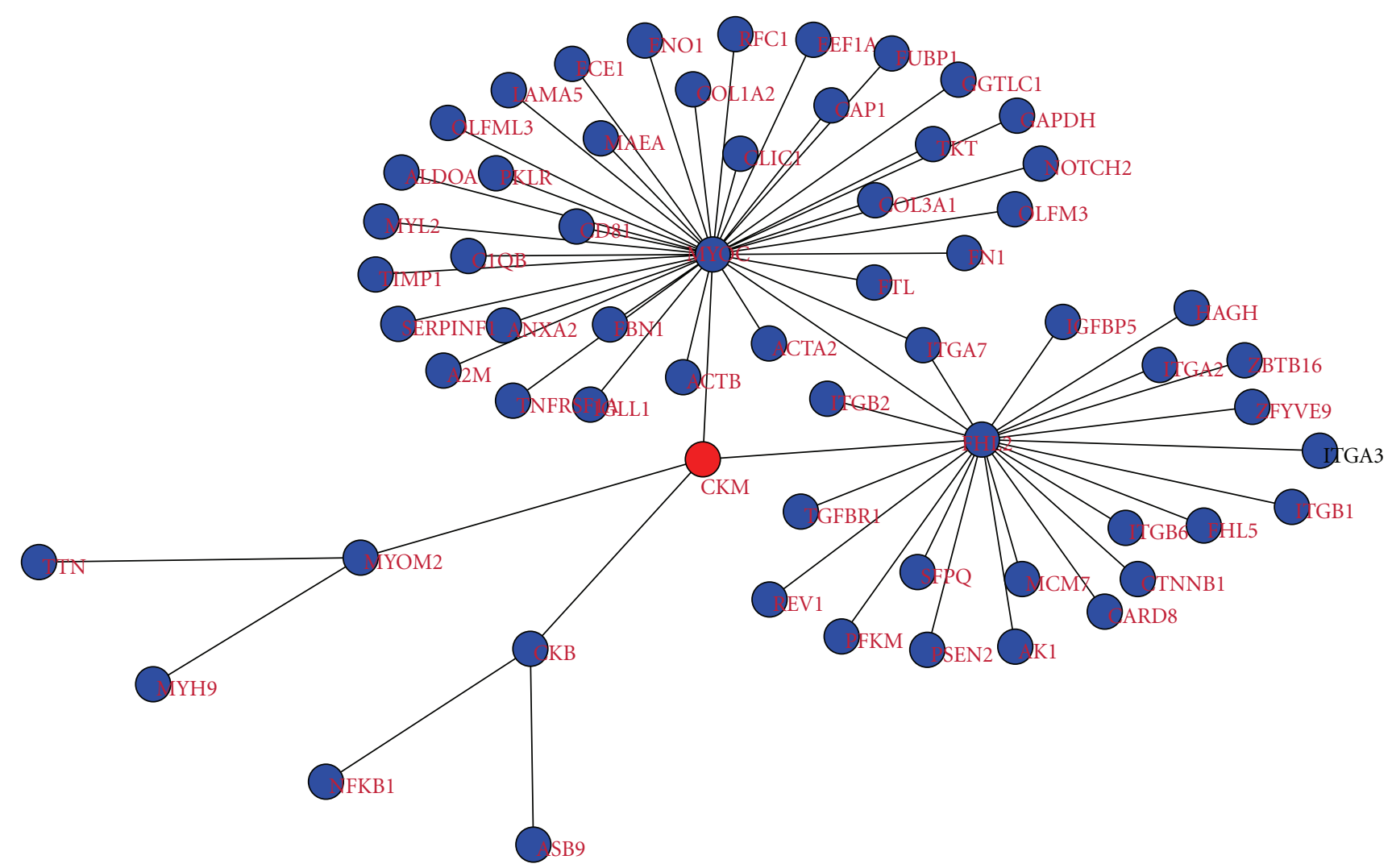

Figure 4: PPI map for CKM as a target hub protein with the $100 \%$ identity. The methodological conditions were the same as for Figure 3 except the identity.

databases. The image was made by the Pajak2.00 program (http://vlado.fmf.uni-lj.si/pub/networks/pajek/).

\section{Results and Discussion}

We identified potential candidates through protein-protein interaction predictions made using various protein interaction resources. By analyzing the hub protein of the networks with metrics such as degree and centrality, we detected 123 potential candidates for $\mathrm{CKB}$ interacting (direct or indirect) factors and 85 candidates for CKM.

In Figure 1, interacting factors such as NFKB1 (NP_003989, nuclear factor of kappa light polypeptide gene enhancer in B-cells 1), MYOC (NP_000252; myocilin, trabecular meshwork inducible glucocorticoid response), MYOM2 (NP_003961; myomesin (M-protein) 2, $165 \mathrm{kDa}$ ), FHL2 (NP_001034581, four-and-a-half LIM domains 2), HIF1AN (NP_060372, hypoxia-inducible factor 1, alpha subunit inhibitor), ASB9 (NP_076992, ankyrin repeat and SOCS box-containing 9), and CKM (NP_001815, creatine kinase, muscle) were elucidated. Interestingly, NFKB1 was detected as a hub protein interacting with CK-BB in our results. In Figure 2, we obtained results similar to those from Figure 1, where NFKB1, MYOC, MYOM2, FHL2, HIF1AN, ASB9, and CKM were detected as interacting factors that were directly or indirectly associated with CKB. NFKB1, CKM, and ASB9 interacted with CKB directly.
In the same way, we detected the CKM-associated proteins as shown in Figure 3 with $80 \%$ sequence identity. As a result, we found that CKB, FHL2, MYOC, ASB9, HIF1AN, NFKB1, TTN (NP_596870, titin), MYH9 (NP_002464, myosin, heavy chain 9, non-muscle), and ITGA7 (NP_002197, integrin, alpha 7) mainly interacted with CKM at $80 \%$ sequence identity. At the level of $100 \%$ identity, we found that MYOM2, CKB, FHL2, and MYOC directly interacted with CKM as shown in Figure 4. In addition to these factors, complete lists of factors that interacted with $\mathrm{CKB}$ and $\mathrm{CKM}$ in a direct or indirect manner are shown in Tables 1 and 2. After overlapping the results from Figures 1 to 4 , we found that NFKB1, FHL2, and MYOC were still detected as hub proteins in Figure 5.

NFKB1 (also known as p50 or NF-kappaB) is a wellknown transcription regulator that is responsible for the expression and regulation of many genes for immune response, cell adhesion, differentiation, proliferation, angiogenesis, and apoptosis [27-31]. It translocates into the nucleus and stimulates the expression of many genes involved in various biological functions. NFKB1 is also associated with a number of inflammatory diseases such as lymphoma [32], Alzheimer disease [33], psoriatic arthritis [34], breast cancer $[35,36]$, and rheumatoid arthritis [37]. Activation of NFKB1 requires binding of NF-kappaB essential modulator (NEMO) to ubiquitinated substrates [38]. With respect to an association with CK, it has been reported that NFKB1 is mostly associated with myocardial ischemia/reperfusion. 
TABLE 1: Gene lists for the analyses of the PEIMAP and PSIMAP using CK-BB as a hub protein with 100\% identity.

\begin{tabular}{|c|c|c|}
\hline Gene ID & Gene symbol & Full name \\
\hline 6256 & RXRA & Retinoid X receptor, alpha \\
\hline 3309 & HSPA5 & Heat shock $70 \mathrm{kDa}$ protein 5 (glucose-regulated protein, $78 \mathrm{kDa}$ ) \\
\hline 3320 & HSP90AA1 & Heat shock protein $90 \mathrm{kDa}$ alpha (cytosolic), class A member 1 \\
\hline 2908 & $\mathrm{NR} 3 \mathrm{C} 1$ & Nuclear receptor subfamily 3 , group C, member 1 (glucocorticoid receptor) \\
\hline 6778 & STAT6 & Signal transducer and activator of transcription 6 , interleukin- 4 induced \\
\hline 3146 & HMGB1 & High-mobility group box 1 \\
\hline 3301 & DNAJA1 & DnaJ (Hsp40) homolog, subfamily A, member 1 \\
\hline 4221 & MEN1 & Multiple endocrine neoplasia I \\
\hline 3312 & HSPA8 & Heat shock $70 \mathrm{kDa}$ protein 8 \\
\hline 3840 & KPNA4 & Karyopherin alpha 4 (importin alpha 3 ) \\
\hline 2274 & FHL2 & Four-and-a-half LIM domains 2 \\
\hline 4792 & NFKBIA & Nuclear factor of kappa light polypeptide gene enhancer in B-cells inhibitor, alpha \\
\hline 57805 & KIAA1967 & KIAA1967 \\
\hline 3185 & HNRNPF & Heterogeneous nuclear ribonucleoprotein $\mathrm{F}$ \\
\hline 203068 & TUBB & Tubulin, beta \\
\hline 6774 & STAT3 & Signal transducer and activator of transcription 3 (acute-phase response factor) \\
\hline 4670 & HNRNPM & Heterogeneous nuclear ribonucleoprotein $\mathrm{M}$ \\
\hline 1997 & ELF1 & E74-like factor 1 (ets domain transcription factor) \\
\hline 113457 & TUBA3D & Tubulin, alpha 3D \\
\hline 1999 & ELF3 & E74-like factor 3 (ets domain transcription factor, epithelial-specific) \\
\hline 5591 & PRKDC & Protein kinase, DNA-activated, catalytic polypeptide \\
\hline 708 & C1QBP & Complement component 1 , q subcomponent binding protein \\
\hline 2274 & FHL2 & Four-and-a-half LIM domains 2 \\
\hline 3313 & HSPA9 & Heat shock $70 \mathrm{kDa}$ protein 9 (mortalin) \\
\hline 8600 & TNFSF11 & Tumor necrosis factor (ligand) superfamily, member 11 \\
\hline 3659 & IRF1 & Interferon regulatory factor 1 \\
\hline 84617 & TUBB6 & Tubulin, beta 6 \\
\hline 7280 & TUBB2A & Tubulin, beta $2 \mathrm{~A}$ \\
\hline 2908 & $\mathrm{NR} 3 \mathrm{C} 1$ & Nuclear receptor subfamily 3 , group C, member 1 (glucocorticoid receptor) \\
\hline 2908 & $\mathrm{NR} 3 \mathrm{C} 1$ & Nuclear receptor subfamily 3 , group C, member 1 (glucocorticoid receptor) \\
\hline 8517 & IKBKG & Inhibitor of kappa light polypeptide gene enhancer in B-cells, kinase gamma \\
\hline 7295 & TXN & Thioredoxin \\
\hline 10318 & TNIP1 & TNFAIP3 interacting protein 1 \\
\hline 4793 & NFKBIB & Nuclear factor of kappa light polypeptide gene enhancer in B-cells inhibitor, beta \\
\hline 3065 & HDAC1 & Histone deacetylase 1 \\
\hline 3551 & IKBKB & inhibitor of kappa light polypeptide gene enhancer in B-cells, kinase beta \\
\hline 1152 & $\mathrm{CKB}$ & Creatine kinase, brain \\
\hline 4069 & LYZ & Lysozyme (renal amyloidosis) \\
\hline 140462 & ASB9 & Ankyrin repeat and SOCS box-containing 9 \\
\hline 4653 & MYOC & Myocilin, trabecular meshwork inducible glucocorticoid response \\
\hline 6774 & STAT3 & Signal transducer and activator of transcription 3 (acute-phase response factor) \\
\hline 3660 & IRF2 & Interferon regulatory factor 2 \\
\hline 7278 & TUBA3C & Tubulin, alpha $3 \mathrm{c}$ \\
\hline 4221 & MEN1 & Multiple endocrine neoplasia I \\
\hline 5966 & REL & v-rel reticuloendotheliosis viral oncogene homolog (avian) \\
\hline 1147 & CHUK & Conserved helix-loop-helix ubiquitous kinase \\
\hline 55922 & NKRF & NFKB repressing factor \\
\hline 2113 & ETS1 & v-ets erythroblastosis virus E26 oncogene homolog 1 (avian) \\
\hline 64332 & NFKBIZ & Nuclear factor of kappa light polypeptide gene enhancer in B-cells inhibitor, zeta \\
\hline
\end{tabular}


Table 1: Continued.

\begin{tabular}{|c|c|c|}
\hline Gene ID & Gene symbol & Full name \\
\hline 51773 & RSF1 & Remodeling and spacing factor 1 \\
\hline 5971 & RELB & v-rel reticuloendotheliosis viral oncogene homolog B \\
\hline 1832 & DSP & Desmoplakin \\
\hline 347733 & TUBB2B & Tubulin, beta $2 \mathrm{~B}$ \\
\hline 2353 & FOS & $\mathrm{v}$-fos FBJ murine osteosarcoma viral oncogene homolog \\
\hline 9325 & TRIP4 & Thyroid hormone receptor interactor 4 \\
\hline 4435 & CITED1 & $\mathrm{Cbp} / \mathrm{p} 300$-interacting transactivator, with Glu/Asp-rich carboxy-terminal domain, 1 \\
\hline 22984 & PDCD11 & Programmed cell death 11 \\
\hline 790 & $\mathrm{CAD}$ & Carbamoyl-phosphate synthetase 2 , aspartate transcarbamylase, and dihydroorotase \\
\hline 1326 & MAP3K8 & Mitogen-activated protein kinase kinase kinase 8 \\
\hline 1917 & EEF1A2 & Eukaryotic translation elongation factor 1 alpha 2 \\
\hline 9172 & MYOM2 & Myomesin (M-protein) 2, $165 \mathrm{kDa}$ \\
\hline 10856 & RUVBL2 & RuvB-like 2 (E. coli) \\
\hline 1158 & CKM & Creatine kinase, muscle \\
\hline 808 & CALM3 & Calmodulin 3 (phosphorylase kinase, delta) \\
\hline 672 & BRCA1 & Breast cancer 1 , early onset \\
\hline 801 & CALM1 & Calmodulin 1 (phosphorylase kinase, delta) \\
\hline 293 & SLC25A6 & Solute carrier family 25 (mitochondrial carrier; adenine nucleotide translocator), member 6 \\
\hline 3310 & HSPA6 & Heat shock $70 \mathrm{kDa}$ protein $6\left(\mathrm{HSP} 70 \mathrm{~B}^{\prime}\right)$ \\
\hline 2908 & NR3C1 & Nuclear receptor subfamily 3, group C, member 1 (glucocorticoid receptor) \\
\hline 136319 & MTPN & Myotrophin \\
\hline 2274 & FHL2 & Four-and-a-half LIM domains 2 \\
\hline 9093 & DNAJA3 & DnaJ (Hsp40) homolog, subfamily A, member 3 \\
\hline 4628 & MYH10 & Myosin, heavy chain 10 , non-muscle \\
\hline 4221 & MEN1 & Multiple endocrine neoplasia I \\
\hline 6774 & STAT3 & Signal transducer and activator of transcription 3 (acute-phase response factor) \\
\hline 3839 & KPNA3 & Karyopherin alpha 3 (importin alpha 4) \\
\hline 57805 & KIAA1967 & KIAA1967 \\
\hline 2908 & $\mathrm{NR} 3 \mathrm{C} 1$ & Nuclear receptor subfamily 3 , group C, member 1 (glucocorticoid receptor) \\
\hline 1869 & $\mathrm{E} 2 \mathrm{~F} 1$ & E2F transcription factor 1 \\
\hline 55662 & HIF1AN & Hypoxia-inducible factor 1 , alpha subunit inhibitor \\
\hline 79155 & TNIP2 & TNFAIP3 interacting protein 2 \\
\hline 9532 & BAG2 & BCL2-associated athanogene 2 \\
\hline 6421 & SFPQ & Splicing factor proline/glutamine-rich (polypyrimidine tract binding protein associated) \\
\hline 2908 & NR3C1 & Nuclear receptor subfamily 3, group C, member 1 (glucocorticoid receptor) \\
\hline 10627 & MRCL3 & Myosin regulatory light chain MRCL3 \\
\hline 7431 & VIM & Vimentin \\
\hline 672 & BRCA1 & Breast cancer 1 , early onset \\
\hline 2274 & FHL2 & Four-and-a-half LIM domains 2 \\
\hline 4221 & MEN1 & Multiple endocrine neoplasia I \\
\hline 672 & BRCA1 & Breast cancer 1 , early onset \\
\hline 4221 & MEN1 & Multiple endocrine neoplasia I \\
\hline 3836 & KPNA1 & Karyopherin alpha 1 (importin alpha 5) \\
\hline 3093 & UBE2K & Ubiquitin-conjugating enzyme E2K (UBC1 homolog, yeast) \\
\hline 805 & CALM2 & Calmodulin 2 (phosphorylase kinase, delta) \\
\hline 5970 & RELA & v-rel reticuloendotheliosis viral oncogene homolog A (avian) \\
\hline 9782 & MATR3 & Matrin 3 \\
\hline 8600 & TNFSF11 & Tumor necrosis factor (ligand) superfamily, member 11 \\
\hline 8607 & RUVBL1 & RuvB-like 1 (E. coli) \\
\hline
\end{tabular}


TABle 1: Continued.

\begin{tabular}{|c|c|c|}
\hline Gene ID & Gene symbol & Full name \\
\hline 4627 & MYH9 & Myosin, heavy chain 9 , nonmuscle \\
\hline 23421 & ITGB3BP & Integrin beta 3 binding protein (beta3-endonexin) \\
\hline 140462 & ASB9 & Ankyrin repeat and SOCS box-containing 9 \\
\hline 4841 & NONO & Non-POU domain containing, octamer-binding \\
\hline 9276 & COPB2 & Coatomer protein complex, subunit beta 2 (beta prime) \\
\hline 4221 & MEN1 & Multiple endocrine neoplasia I \\
\hline 1213 & CLTC & Clathrin, heavy chain (Hc) \\
\hline 292 & SLC25A5 & Solute carrier family 25 (Mitochondrial carrier; adenine nucleotide translocator), member 5 \\
\hline 4066 & LYL1 & Lymphoblastic leukemia-derived sequence 1 \\
\hline 64332 & NFKBIZ & Nuclear factor of kappa light polypeptide gene enhancer in B-cells inhibitor, zeta \\
\hline 5531 & PPP4C & Protein phosphatase 4 (formerly X), catalytic subunit \\
\hline 8091 & HMGA2 & High-mobility group AT-hook 2 \\
\hline 6202 & RPS8 & Ribosomal protein S8 \\
\hline 1051 & CEBPB & CCAAT/enhancer binding protein $(\mathrm{C} / \mathrm{EBP})$, beta \\
\hline 222643 & UNC5CL & Unc-5 homolog C (C. elegans)-like \\
\hline 4790 & NFKB1 & Nuclear factor of kappa light polypeptide gene enhancer in B-cells 1 \\
\hline 71 & ACTG1 & Actin, gamma 1 \\
\hline 3312 & HSPA8 & Heat shock $70 \mathrm{kDa}$ protein 8 \\
\hline 9782 & MATR3 & Matrin 3 \\
\hline 3320 & HSP90AA1 & Heat shock protein $90 \mathrm{kDa}$ alpha (cytosolic), class A member 1 \\
\hline 4637 & MYL6 & Myosin, light chain 6, alkali, smooth muscle and nonmuscle \\
\hline 2908 & $\mathrm{NR} 3 \mathrm{C} 1$ & Nuclear receptor subfamily 3 , group C, member 1 (glucocorticoid receptor) \\
\hline 4793 & NFKBIB & Nuclear factor of kappa light polypeptide gene enhancer in B-cells inhibitor, beta \\
\hline 688 & KLF5 & Kruppel-like factor 5 (intestinal) \\
\hline 672 & BRCA1 & Breast cancer 1 , early onset \\
\hline
\end{tabular}

During reperfusion, the absence of poly(ADP-ribose) polymerase-1 (PARP-1) leads to a reduction of myocardial apoptosis, which is associated with reduced NFKB1 activation $[39,40]$, and proteasome inhibition ablates activation of NFKB1 in myocardial reperfusion and reduces reperfusion injury [41]. Myocardial injury was assessed by measuring the serum levels of CK, and CK was reduced in serum along with reduction of NFKB1 activation.

FHL2 is a member of the human four-and-a-half-LIMonly protein family, which consists of the members FHL1, FHL2, FHL3, FHL4, and ACT. These proteins function in various cellular processes, including regulation of cell survival, transcription, and signal transduction [42]. FHL2 contains an LIM domain, one of the protein-protein interaction motifs, which allows specific proteins to combine with certain partners. The specificity of a protein-protein interaction can be obtained by an interaction code predicted by conserved amino acid sequences. The interaction of FHL2 with transcription factors and other proteins involved in cancer development was examined. Since transcription factors control all fundamental developmental and homeostatic processes, transcriptional cofactors such as FHL2 are likely to contribute to human carcinogenesis and are of clinical importance in various forms of cancer [43], including leukemia [44]. With respect to an association with CK, Chung et al. [45] reported that FHL2 (developmentally enhanced phosphotransfer enzyme-anchoring protein) amalgamated the myofibrillar $\mathrm{CK}$ metabolic signaling circuit, providing an energetic continuum between mitochondria and the nascent contractile machinery in a murine embryonic stem cell cardiac differentiation model. They reported that CK$\mathrm{M}$ clustered around developing myofibrils, sarcolemma, and the perinuclear compartment, whereas CK-B was tightly associated with myofibrillar alpha-actinin, forming wirelike structures extending from the nuclear compartment to the sarcolemma. FHL2 was also increased in myocardial ischemia-reperfusion injury, where IL- 6 and IL- 8 mRNA are upregulated in human cardiac myocytes [46].

Recently, ASB9 was found to interact with ubiquitous mitochondrial CK [47]. The ankyrin repeat domains of ASB9 can associate with the substrate binding site of CK in a SOCS box-independent manner. The overexpression of ASB9 induces ubiquitination of CK. ASB9 reduces CK activities and cell growth and negatively regulates cell growth. ASB9 is a member of the ankyrin repeat and is a suppressor of the cytokine signaling (SOCS) box protein family. It can interact with the SOCS box domain of the elongin B-C adapter complex and can further complex with the cullin and ring box proteins to form E3 ubiquitin ligase complexes [48]. These complexes may be involved in specific substraterecognition for ubiquitination and degradation and mediate the substrate-recognition of the E3 ubiquitin ligases. 
TABLE 2: Gene lists for the analyses of the PEIMAP and PSIMAP using CK-MM as a hub protein with 100\% identity.

\begin{tabular}{|c|c|c|}
\hline Gene ID & Gene symbol & Full name \\
\hline 1889 & ECE1 & Endothelin-converting enzyme 1 \\
\hline 5981 & RFC1 & Replication factor C (activator 1) 1, $145 \mathrm{kDa}$ \\
\hline 226 & ALDOA & Aldolase A, fructose-bisphosphate \\
\hline 2335 & FN1 & Fibronectin 1 \\
\hline 9372 & ZFYVE9 & Zinc finger, FYVE domain containing 9 \\
\hline 60 & ACTB & Actin, beta \\
\hline 3688 & ITGB1 & Integrin, beta 1 (fibronectin receptor, beta polypeptide, antigen CD29 includes MDF2, MSK12) \\
\hline 7273 & TTN & Titin \\
\hline 2274 & FHL2 & Four-and-a-half LIM domains 2 \\
\hline 4853 & NOTCH2 & Notch homolog 2 (Drosophila) \\
\hline 2512 & FTL & Ferritin, light polypeptide \\
\hline 1192 & CLIC1 & Chloride intracellular channel 1 \\
\hline 2274 & FHL2 & Four-and-a-half LIM domains 2 \\
\hline 5313 & PKLR & Pyruvate kinase, liver and RBC \\
\hline 302 & ANXA2 & Annexin A2 \\
\hline 7704 & ZBTB16 & Zinc finger and BTB domain containing 16 \\
\hline 2200 & FBN1 & Fibrillin 1 \\
\hline 27332 & ZNF638 & Zinc finger protein 638 \\
\hline 92086 & GGTLC1 & Gamma-glutamyltransferase light chain 1 \\
\hline 713 & C1QB & Complement component 1 , q subcomponent, B chain \\
\hline 3029 & HAGH & Hydroxyacylglutathione hydrolase \\
\hline 5664 & PSEN2 & Presenilin 2 (Alzheimer disease 4) \\
\hline 7086 & TKT & Transketolase (Wernicke-Korsakoff syndrome) \\
\hline 4176 & MCM7 & Minichromosome maintenance complex component 7 \\
\hline 1152 & $\mathrm{CKB}$ & Creatine kinase, brain \\
\hline 1499 & CTNNB1 & Catenin (cadherin-associated protein), beta $1,88 \mathrm{kDa}$ \\
\hline 140462 & ASB9 & Ankyrin repeat and SOCS box-containing 9 \\
\hline 9457 & FHL5 & Four-and-a-half LIM domains 5 \\
\hline 4653 & MYOC & Myocilin, trabecular meshwork inducible glucocorticoid response \\
\hline 3029 & $\mathrm{HAGH}$ & Hydroxyacylglutathione hydrolase \\
\hline 7704 & ZBTB16 & Zinc finger and BTB domain containing 16 \\
\hline 3675 & ITGA3 & Integrin, alpha 3 (antigen CD49C, alpha 3 subunit of VLA-3 receptor) \\
\hline 226 & ALDOA & Aldolase A, fructose-bisphosphate \\
\hline 3689 & ITGB2 & Integrin, beta 2 (complement component 3 receptor 3 and 4 subunit) \\
\hline 3688 & ITGB1 & Integrin, beta 1 (fibronectin receptor, beta polypeptide, antigen CD29 includes MDF2, MSK12) \\
\hline 302 & ANXA2 & Annexin A2 \\
\hline 92086 & GGTLC1 & Gamma-glutamyltransferase light chain 1 \\
\hline 3679 & ITGA7 & Integrin, alpha 7 \\
\hline 2023 & ENO1 & Enolase 1, (alpha) \\
\hline 9172 & MYOM2 & Myomesin (M-protein) 2, $165 \mathrm{kDa}$ \\
\hline 1158 & CKM & Creatine kinase, muscle \\
\hline 4790 & NFKB1 & Nuclear factor of kappa light polypeptide gene enhancer in B-cells 1 (p105) \\
\hline 2 & $\mathrm{~A} 2 \mathrm{M}$ & Alpha-2-macroglobulin \\
\hline 3688 & ITGB1 & Integrin, beta 1 (fibronectin receptor, beta polypeptide, antigen CD29 includes MDF2, MSK12) \\
\hline 56944 & OLFML3 & Olfactomedin-like 3 \\
\hline 1281 & COL3A1 & Collagen, type III, alpha 1 (Ehlers-Danlos syndrome type IV, autosomal dominant) \\
\hline 2274 & FHL2 & Four-and-a-half LIM domains 2 \\
\hline 3688 & ITGB1 & Integrin, beta 1 (fibronectin receptor, beta polypeptide, antigen CD29 includes MDF2, MSK12) \\
\hline
\end{tabular}


TABle 2: Continued.

\begin{tabular}{|c|c|c|}
\hline Gene ID & Gene symbol & Full name \\
\hline 118427 & OLFM3 & Olfactomedin 3 \\
\hline 22900 & CARD8 & Caspase recruitment domain family, member 8 \\
\hline 3488 & IGFBP5 & Insulin-like growth factor binding protein 5 \\
\hline 7132 & TNFRSF1A & Tumor necrosis factor receptor superfamily, member $1 \mathrm{~A}$ \\
\hline 226 & ALDOA & Aldolase A, fructose-bisphosphate \\
\hline 3675 & ITGA3 & Integrin, alpha 3 (antigen CD49C, alpha 3 subunit of VLA-3 receptor) \\
\hline 51455 & REV1 & REV1 homolog (S. cerevisiae) \\
\hline 6421 & SFPQ & Splicing factor proline/glutamine-rich (polypyrimidine tract binding protein associated) \\
\hline 302 & ANXA2 & Annexin A2 \\
\hline 4633 & MYL2 & Myosin, light chain 2, regulatory, cardiac, slow \\
\hline 8880 & FUBP1 & Far upstream element (FUSE) binding protein 1 \\
\hline 2274 & FHL2 & Four-and-a-half LIM domains 2 \\
\hline 3688 & ITGB1 & Integrin, beta 1 (fibronectin receptor, beta polypeptide, antigen CD29 includes MDF2, MSK12) \\
\hline 27332 & ZNF638 & Zinc finger protein 638 \\
\hline 3673 & ITGA2 & Integrin, alpha 2 (CD49B, alpha 2 subunit of VLA-2 receptor) \\
\hline 203 & AK1 & Adenylate kinase 1 \\
\hline 4627 & MYH9 & Myosin, heavy chain 9 , non-muscle \\
\hline 5213 & PFKM & Phosphofructokinase, muscle \\
\hline 140462 & ASB9 & Ankyrin repeat and SOCS box-containing 9 \\
\hline 7076 & TIMP1 & TIMP metallopeptidase inhibitor 1 \\
\hline 5176 & SERPINF1 & $\begin{array}{l}\text { Serpin peptidase inhibitor, clade F (alpha-2 antiplasmin, pigment epithelium derived factor), } \\
\text { member } 1\end{array}$ \\
\hline 3694 & ITGB6 & Integrin, beta 6 \\
\hline 59 & ACTA2 & Actin, alpha 2, smooth muscle, aorta \\
\hline 4176 & MCM7 & Minichromosome maintenance complex component 7 \\
\hline 10487 & CAP1 & CAP, adenylate cyclase-associated protein 1 (yeast) \\
\hline 7046 & TGFBR1 & Transforming growth factor, beta receptor I (activin A receptor type II-like kinase, $53 \mathrm{kDa}$ ) \\
\hline 3543 & IGLL1 & Immunoglobulin lambda-like polypeptide 1 \\
\hline 5313 & PKLR & Pyruvate kinase, liver and RBC \\
\hline 51455 & REV1 & REV1 homolog (S. cerevisiae) \\
\hline 10296 & MAEA & Macrophage erythroblast attacher \\
\hline 3911 & LAMA5 & Laminin, alpha 5 \\
\hline 2597 & GAPDH & Glyceraldehyde-3-phosphate dehydrogenase \\
\hline 975 & CD81 & CD81 molecule \\
\hline 92086 & GGTLC1 & Gamma-glutamyltransferase light chain 1 \\
\hline 1915 & EEF1A1 & Eukaryotic translation elongation factor 1 alpha 1 \\
\hline 5664 & PSEN2 & Presenilin 2 (Alzheimer disease 4) \\
\hline 1278 & COL1A2 & Collagen, type I, alpha 2 \\
\hline
\end{tabular}

The interaction between CK and MYOC has not been elucidated. However, MYOC has a cytoskeletal function, and this implies that it may interact with CK somehow. MYOC is expressed in many ocular tissues including the trabecular meshwork [49], which is a specialized eye tissue that is essential in regulating intraocular pressure. MYOC mutations have been identified as the cause of hereditary juvenile-onset open-angle glaucoma [50].

Researchers could apply computational prediction by PPI mapping to help determine target proteins. Since the next step in the functional study of interesting proteins/genes is a time- and cost-consuming process, the number of target proteins is limited; hence, for the right choice, computational prediction on the basis of database information could be critical at this step. Functional studies can be further conducted using a mouse model and a large number of clinical samples. Final confirmation and CK mechanisms could then be more clearly evaluated for developing drugs to effectively treat CK-related diseases.

The functions of most of the candidate proteins predicted in this study have not been well reported in skin diseases or in the pathogenesis of other diseases. We provide new 


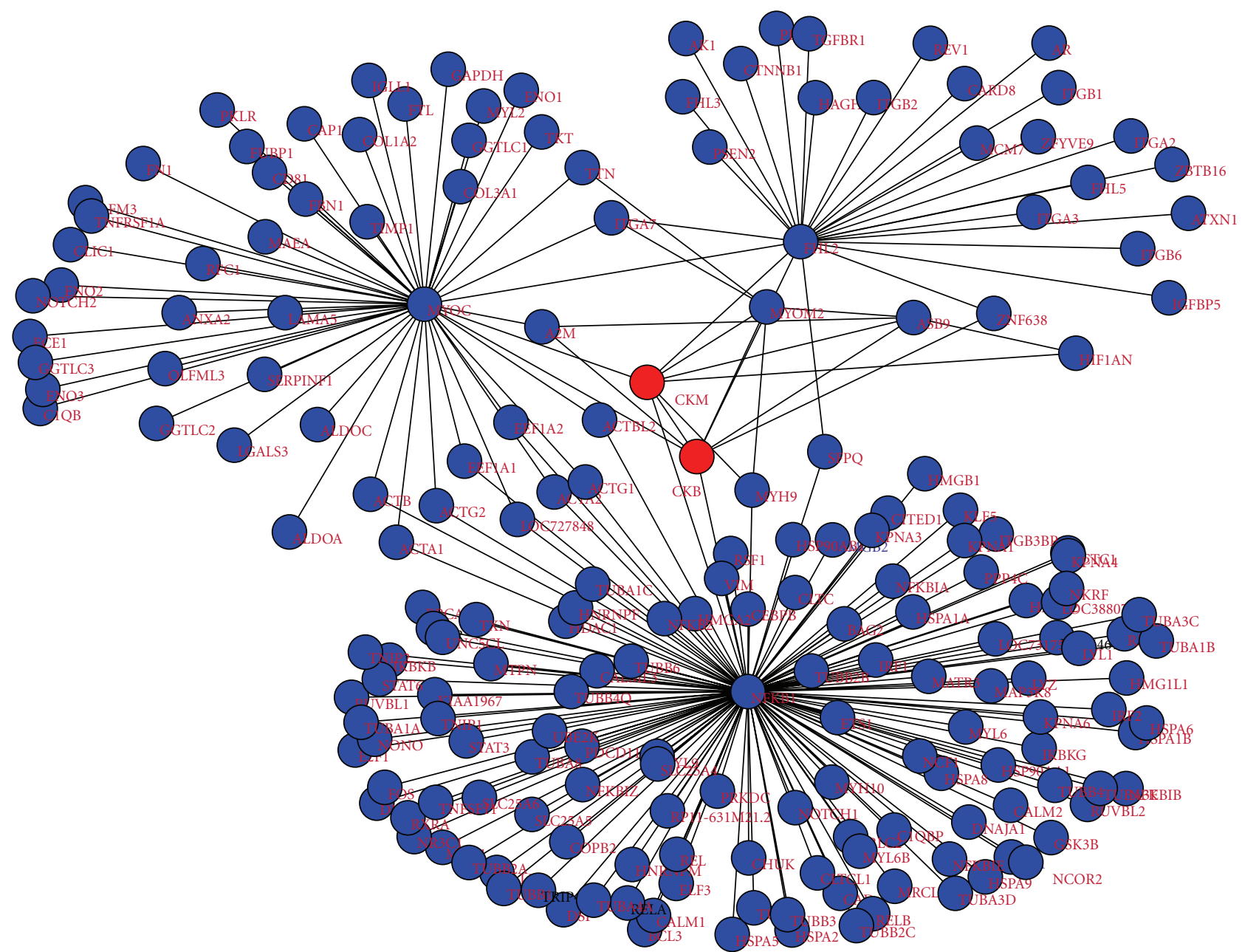

Figure 5: Overlapping map between CKB and CKM PPI maps. Data were input by using the results from Figures 1 to 4 .

information regarding these candidate proteins' interaction with $\mathrm{CK}$, as well as the involvement of several hub proteins such as NFKB1, FHL2, ASB9, and MYOC. Although we do not suggest a direct role of any candidate protein in skin diseases, we provide candidate proteins to be targeted in further studies of CK-associated diagnostic markers and/or treatment of corresponding skin conditions. Furthermore, we also provide some insights into understanding the responses of CK in skin.

\section{Abbreviations}

PPI: $\quad$ Protein-protein interaction

CK-MM: Muscle type homodimer

CK-BB: Brain type homodimer.

\section{Acknowledgments}

This study was supported by the Grants from the Science and Technology Bureau of Jiaxing, Zhejiang (no. 2008AY2032) and the Science and Technology Planning Project of Zhejiang Province (no. 2010C33139). Dr. W.-J. Hu was supported by a Grant from China Postdoctoral Science Foundation (no. 20060400467). Dr. S.-M. Zhou was supported by a grant from the Science and Technology Bureau of Jiaxing (no. 2007AY2021).

\section{References}

[1] U. Schlattner, M. Forstner, M. Eder, O. Stachowiak, K. FritzWolf, and T. Wallimann, "Functional aspects of the Xray structure of mitochondrial creatine kinase: a molecular physiology approach," Molecular and Cellular Biochemistry, vol. 184, no. 1-2, pp. 125-140, 1998.

[2] M. J. McLeish and G. L. Kenyon, "Relating structure to mechanism in creatine kinase," Critical Reviews in Biochemistry and Molecular Biology, vol. 40, no. 1, pp. 1-20, 2005.

[3] D. R. Abendschein, "Rapid diagnosis of myocardial infarction and reperfusion by assay of plasma isoforms of creatine kinase isoenzymes," Clinical Biochemistry, vol. 23, no. 5, pp. 399-407, 1990.

[4] S. H. Smith, M. F. Kramer, I. Reis, S. P. Bishop, and J. S. Ingwall, "Regional changes in creatine kinase and myocyte size in hypertensive and nonhypertensive cardiac hypertrophy," Circulation Research, vol. 67, no. 6, pp. 1334-1344, 1990. 
[5] E. Ozawa, Y. Hagiwara, and M. Yoshida, "Creatine kinase, cell membrane and Duchenne muscular dystrophy," Molecular and Cellular Biochemistry, vol. 190, no. 1-2, pp. 143-151, 1999.

[6] T. S. Bürklen, U. Schlattner, R. Homayouni et al., "The creatine kinase/creatine connection to alzheimer's disease: CKinactivation, APP-CK complexes and focal creatine deposits," Journal of Biomedicine and Biotechnology, vol. 2006, no. 3, Article ID 35936, 11 pages, 2006.

[7] H. Takubo, S. Shimoda-Matsubayashi, and Y. Mizuno, "Serum creatine kinase is elevated in patients with Parkinson's disease: a case controlled study," Parkinsonism and Related Disorders, vol. 9, supplement 1, pp. S43-S46, 2003.

[8] U. Schlattner, N. Möckli, O. Speer, S. Werner, and T. Wallimann, "Creatine kinase and creatine transporter in normal, wounded, and diseased skin," Journal of Investigative Dermatology, vol. 118, no. 3, pp. 416-423, 2002.

[9] E. J. Chang, J. Ha, F. Oerlemans et al., "Brain-type creatine kinase has a crucial role in osteoclast-mediated bone resorption," Nature Medicine, vol. 14, no. 9, pp. 966-972, 2008.

[10] A. Salin-Cantegrel, M. Shekarabi, S. Holbert et al., "HMSN/ACC truncation mutations disrupt brain-type creatine kinase-dependant activation of $\mathrm{K}^{+} / \mathrm{Cl}^{-}$co-transporter 3," Human Molecular Genetics, vol. 17, no. 17, pp. 2703-2711, 2008.

[11] A. Castegna, M. Aksenov, M. Aksenova et al., "Proteomic identification of oxidatively modified proteins in Alzheimer's disease brain. Part I: creatine kinase BB, glutamine synthase, and ubiquitin carboxy-terminal hydrolase L-1," Free Radical Biology and Medicine, vol. 33, no. 4, pp. 562-571, 2002.

[12] M. Aksenov, M. Aksenova, D. A. Butterfield, and W. R. Markesbery, "Oxidative modification of creatine kinase BB in Alzheimer's disease brain," Journal of Neurochemistry, vol. 74, no. 6, pp. 2520-2527, 2000.

[13] J. K. Rao, G. Bujacz, and A. Wlodawer, "Crystal structure of rabbit muscle creatine kinase," FEBS Letters, vol. 439, no. 1-2, pp. 133-137, 1998.

[14] H. M. Zhou and C. L. Tsou, "Comparison of activity and conformation changes during refolding of urea-denatured creatine kinase," Biochimica et Biophysica Acta, vol. 869, no. 1, pp. 69-74, 1986.

[15] Y. D. Park, W. B. Ou, T. W. Yu, and H. M. Zhou, "Folding pathway for partially folded rabbit muscle creatine kinase," Biochemistry and Cell Biology, vol. 79, no. 4, pp. 479-487, 2001.

[16] Y. D. Park, Z. F. Cao, and H. M. Zhou, "Reactivation kinetics of guanidine hydrochloride-denatured creatine kinase measured using the substrate reaction," Journal of Protein Chemistry, vol. 20, no. 1, pp. 67-72, 2001.

[17] S. Li, J. H. Bai, Y. D. Park, and H. M. Zhou, "Capture of monomeric refolding intermediate of human muscle creatine kinase," Protein Science, vol. 15, no. 1, pp. 171-181, 2006.

[18] W. B. Ou, W. Luo, Y. D. Park, and H. M. Zhou, "Chaperonelike activity of peptidyl-prolyl cis-trans isomerase during creatine kinase refolding," Protein Science, vol. 10, no. 11, pp. 2346-2353, 2001.

[19] I. Xenarios, D. W. Rice, L. Salwinski, M. K. Baron, E. M. Marcotte, and D. Eisenberg, "DIP: the database of interacting proteins," Nucleic Acids Research, vol. 28, no. 1, pp. 289-291, 2000.

[20] G. D. Bader, I. Donaldson, C. Wolting, B. F. Ouellette, T. Pawson, and C. W. Hogue, "BIND-the biomolecular interaction network database," Nucleic Acids Research, vol. 29, no. 1, pp. 242-245, 2001.

[21] H. Hermjakob, L. Montecchi-Palazzi, C. Lewington et al., "IntAct: an open source molecular interaction database," Nucleic Acids Research, vol. 32, pp. D452-D455, 2004.
[22] A. Zanzoni, L. Montecchi-Palazzi, M. Quondam, G. Ausiello, M. Helmer-Citterich, and G. Cesareni, "MINT: a molecular INTeraction database," FEBS Letters, vol. 513, no. 1, pp. 135140, 2002.

[23] S. Peri, J. D. Navarro, T. Z. Kristiansen et al., "Human protein reference database as a discovery resource for proteomics," Nucleic Acids Research, vol. 32, pp. D497-D501, 2004.

[24] C. Stark, B. J. Breitkreutz, T. Reguly, L. Boucher, A. Breitkreutz, and M. Tyers, "BioGRID: a general repository for interaction datasets," Nucleic Acids Research, vol. 34, pp. D535-D539, 2006.

[25] A. G. Murzin, S. E. Brenner, T. Hubbard, and C. Chothia, "SCOP: a structural classification of proteins database for the investigation of sequences and structures," Journal of Molecular Biology, vol. 247, no. 4, pp. 536-540, 1995.

[26] J. Park, M. Lappe, and S. A. Teichmann, "Mapping protein family interactions: intramolecular and intermolecular protein family interaction repertoires in the PDB and yeast," Journal of Molecular Biology, vol. 307, no. 3, pp. 929-938, 2001.

[27] X. F. Sun and H. Zhang, "NFKB and NFKBI polymorphisms in relation to susceptibility of tumour and other diseases," Histology and Histopathology, vol. 22, no. 12, pp. 1387-1398, 2007.

[28] S. Patel and D. Santani, "Role of NF- $\kappa$ B in the pathogenesis of diabetes and its associated complications," Pharmacological Reports, vol. 61, no. 4, pp. 595-603, 2009.

[29] L. Verstrepen, I. Carpentier, K. Verhelst, and R. Beyaert, "ABINs: a 20 binding inhibitors of NF- $\kappa \mathrm{B}$ and apoptosis signaling," Biochemical Pharmacology, vol. 78, no. 2, pp. 105$114,2009$.

[30] S. P. Tabruyn and A. W. Griffioen, "NF- $\kappa$ B: a new player in angiostatic therapy," Angiogenesis, vol. 11, no. 1, pp. 101-106, 2008.

[31] T. Okamoto, T. Sanda, and K. Asamitsu, "NK- $\kappa$ B signaling and carcinogenesis," Current Pharmaceutical Design, vol. 13, no. 5, pp. 447-462, 2007.

[32] Q. Qiao, Y. Nozaki, K. Sakoe, N. Komatsu, and K. Kirito, "NF- $\kappa$ B mediates aberrant activation of HIF-1 in malignant lymphoma," Experimental Hematology, vol. 38, no. 12, pp. 1199-1208, 2010.

[33] J. G. Cui, Y. Y. Li, Y. Zhao, S. Bhattacharjee, and W. J. Lukiw, "Differential regulation of interleukin-1 receptor-associated kinase-1 (IRAK-1) and IRAK-2 by microRNA-146a and NF$\kappa \mathrm{B}$ in stressed human astroglial cells and in Alzheimer disease," Journal of Biological Chemistry, vol. 285, no. 50, pp. 3895138960, 2010.

[34] C. Butt, S. Sun, L. Peddle et al., "Association of nuclear factor$\kappa \mathrm{B}$ in psoriatic arthritis," Journal of Rheumatology, vol. 32, no. 9, pp. 1742-1744, 2005.

[35] S. J. van Laere, I. van der Auwera, G. G. van den Eynden et al., "Nuclear factor- $\kappa \mathrm{B}$ signature of inflammatory breast cancer by cDNA microarray validated by quantitative realtime reverse transcription-PCR, immunohistochemistry, and nuclear factor- $\kappa \mathrm{B}$ DNA-binding," Clinical Cancer Research, vol. 12, no. 11, pp. 3249-3256, 2006.

[36] F. Lerebours, S. Vacher, C. Andrieu et al., "NF- $\kappa$ B genes have a major role in inflammatory breast cancer," BMC Cancer, vol. 8, p. 41, 2008.

[37] S. S. Makarov, "NF- $\kappa$ B in rheumatoid arthritis: a pivotal regulator of inflammation, hyperplasia, and tissue destruction," Arthritis Research, vol. 3, no. 4, pp. 200-206, 2001.

[38] S. Rahighi, F. Ikeda, M. Kawasaki et al., "Specific recognition of linear ubiquitin chains by NEMO is important for NF- $\kappa \mathrm{B}$ activation," Cell, vol. 136, no. 6, pp. 1098-1109, 2009. 
[39] B. Zingarelli, P. W. Hake, M. O’Connor, A. Denenberg, S. Kong, and B. J. Aronow, "Absence of poly(ADP-ribose)polymerase- 1 alters nuclear factor- $\kappa$ B activation and gene expression of apoptosis regulators after reperfusion injury," Molecular Medicine, vol. 9, no. 5-8, pp. 143-153, 2003.

[40] J. Yang, J. J. Marden, C. Fan et al., "Genetic redox preconditioning differentially modulates $\mathrm{AP}-1$ and $\mathrm{NF} \kappa \mathrm{B}$ responses following cardiac ischemia/reperfusion injury and protects against necrosis and apoptosis," Molecular Therapy, vol. 7, no. 3, pp. 341-353, 2003.

[41] J. Pye, F. Ardeshirpour, A. McCain et al., "Proteasome inhibition ablates activation of NF- $\kappa$ B in myocardial reperfusion and reduces reperfusion injury," American Journal of Physiology, vol. 284, no. 3, pp. H919-H926, 2003.

[42] M. Johannessen, S. Møler, T. Hansen, U. Moens, and M. van Ghelue, "The multifunctional roles of the four-and-a-halfLIM only protein FHL2," Cellular and Molecular Life Sciences, vol. 63, no. 3, pp. 268-284, 2006.

[43] K. Kleiber, K. Strebhardt, and B. T. Martin, "The biological relevance of FHL2 in tumour cells and its role as a putative cancer target," Anticancer Research, vol. 27, no. 1 A, pp. 55-61, 2007.

[44] Z. Qian, J. M. Joslin, T. R. Tennant et al., "Cytogenetic and genetic pathways in therapy-related acute myeloid leukemia," Chemico Biological Interactions, vol. 184, no. 1-2, pp. 50-57, 2010.

[45] S. Chung, P. P. Dzeja, R. S. Faustino, and A. Terzic, "Developmental restructuring of the creatine kinase system integrates mitochondrial energetics with stem cell cardiogenesis," Annals of the New York Academy of Sciences, vol. 1147, pp. 254-263, 2008.

[46] S. Wan, A. P. Yim, C. K. Wong et al., "Expression of FHL2 and cytokine messenger RNAs in human myocardium after cardiopulmonary bypass," International Journal of Cardiology, vol. 86, no. 2-3, pp. 265-272, 2002.

[47] S. Kwon, D. Kim, J. W. Rhee et al., "ASB9 interacts with ubiquitous mitochondrial creatine kinase and inhibits mitochondrial function," BMC Biology, vol. 8, p. 23, 2010.

[48] X. Fei, Y. Zhang, X. Gu, R. Qiu, Y. Mao, and C. Ji, "Crystallization and preliminary $\mathrm{X}$-ray analysis of the splice variant of human ankyrin repeat and suppressor of cytokine signaling box protein 9 (hASB9-2)," Protein and Peptide Letters, vol. 16, no. 3, pp. 333-335, 2009.

[49] M. K. Joe, S. Sohn, T. E. Kim, J. E. Im, Y. R. Choi, and C. Kee, "Analysis of glucocorticoid-induced MYOC expression in human trabecular meshwork cells," Vision Research, vol. 51, no. 9, pp. 1033-1038, 2011.

[50] X. Zhao, C. Yang, Y. Tong, X. Zhang, L. Xu, and Y. Li, "Identification a novel MYOC gene mutation in a Chinese family with juvenile-onset open angle glaucoma," Molecular Vision, vol. 16, pp. 1728-1735, 2010. 

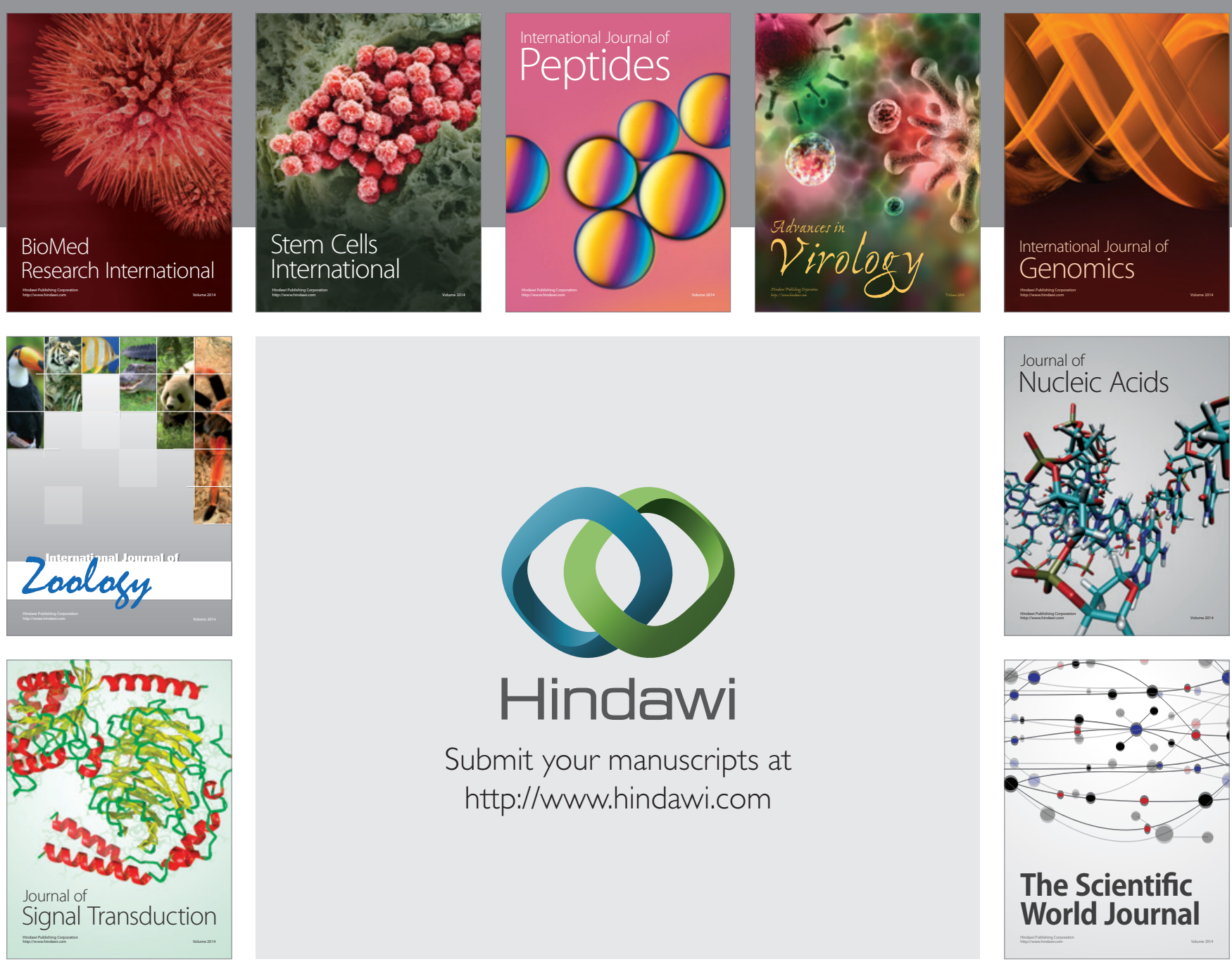

Submit your manuscripts at

http://www.hindawi.com
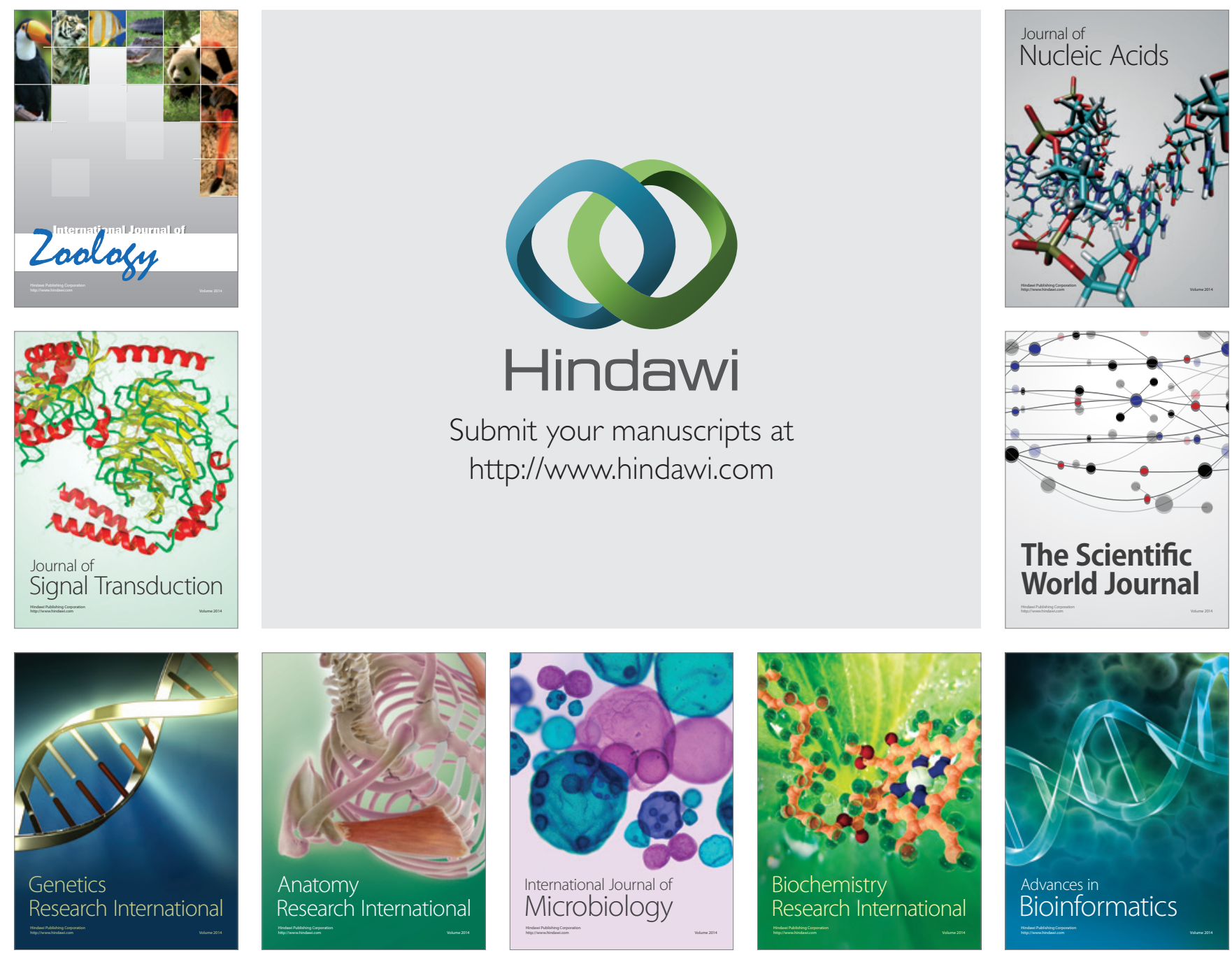

The Scientific World Journal
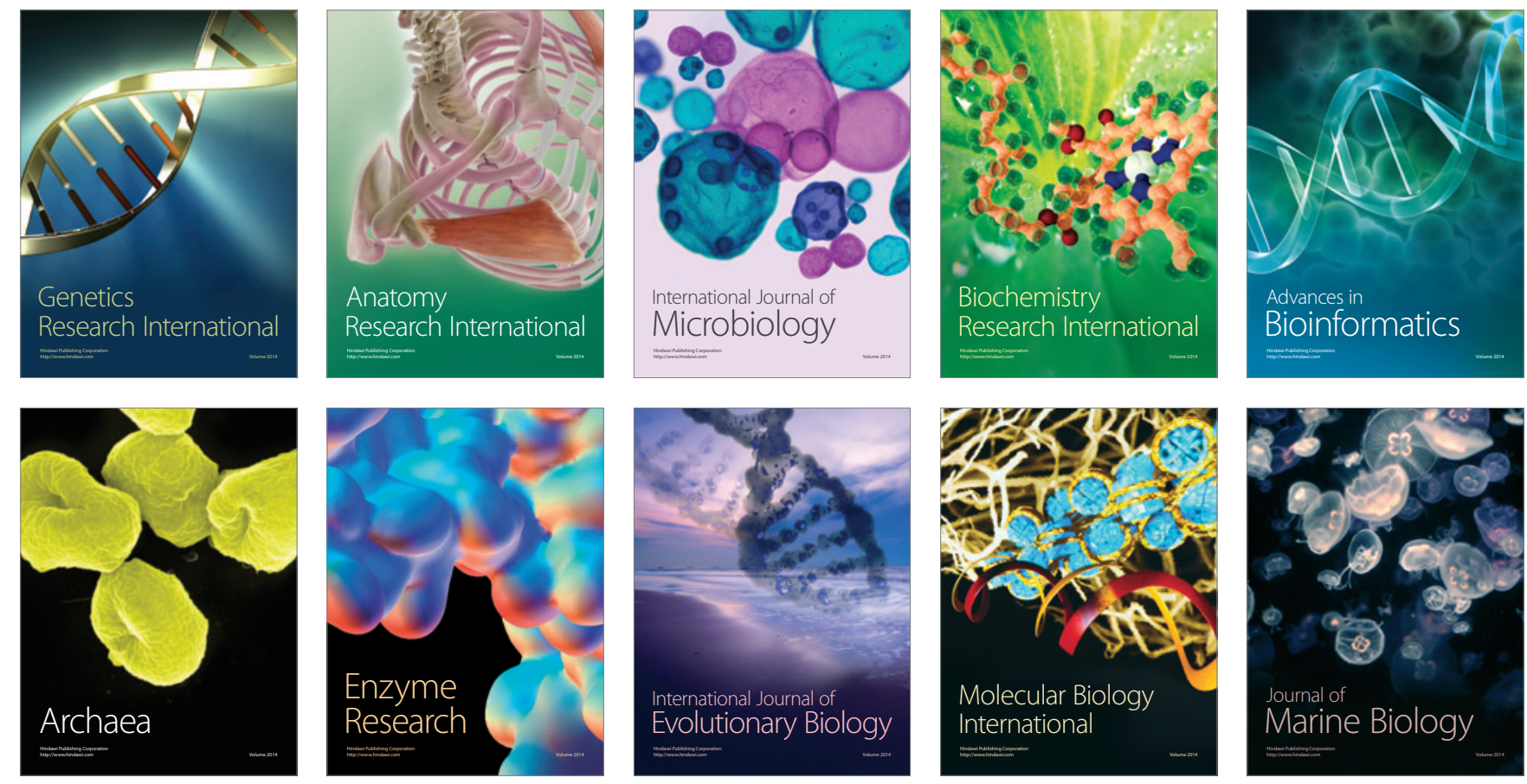